\title{
On the Chinese Network Art Educa- tion in the New Media Era
}

\author{
Xiuli Ma' ${ }^{1}$ Zhanyin $\mathrm{Ma}^{1}$ Bangfan $\mathrm{Liu}^{2}$ Shui $\mathrm{Xu}^{3}$ Haixia Mao ${ }^{1}$ \\ Yongkui Liu ${ }^{3}$ \\ ${ }^{1}$ Hebei Normal University of Science and Technology Qinhuangdao China \\ ${ }^{2}$ Humanities-law College Yanshan University Qinhuangdao China \\ ${ }^{3}$ Qinhuangdao Vocational and Technical College Qinhuangdao China \\ liubangfan@yeah.net
}

\begin{abstract}
With the development of new media the network art will develop rapidly. On one hand, we should vigorously carry out the network art education theory research. In order to carry out the theory research of network art education, we need to comb clearly how can the network art production and dissemination continue according to the healthy and fast road, forming the similar status and value with traditional art form and thus enriching and extending the new space of human spiritual life, under the condition of the Internet media and other new media. On the other hand, we should vigorously carry out the practice and application of network art education. At present, we should pay attention to the following points in the practice and application of network art education: realizing the cultural value of network via culture communication; constructing educational system of the network art through the school teaching; realizing the innovation of the traditional art education through the network art education.
\end{abstract}

Keywords: network art ; new media; art education

\section{Introduction}

Currently, the extension of the concept of "new media" generally includes internet, digital TV, mobile television, mobile phone, IPTV and other media. Some people also think that the new media is a personal media or cross media. Therefore, the new media era has several characteristics: the timeliness of content generation, content obtain at any time, interaction of dissemination, the directional content delivery and so on. In fact, the new media era is the extension of the concepts of the information era and the knowledge era. From the point of view of the development of information science and technology and the popularization of network, we had entered the new media era.

\section{The network art}

The network art concept can be defined in the two aspects of broad sense and narrow sense:

In the broad sense, the so-called " network art"is all the formal art which is connected with the network, or the art which performs through the network, or the are designed by the network technology. That is it not only includes the art in the net, but also includes the art of net; not only includes the sounds, pictures, cartoon network and video network which can be transmitted over the network and processed by digital technology, but also includes treating some network survival condition, or the so-called "net- 
work culture" as the object of expression, but its form of expression may also be the art which is performed by the traditional art on the contrary to the " network multimedia art". ${ }^{[1]}$

In the narrow sense, the so-called "network art" must be the art which transfers and realizes in the net and is proceeded by the digital technology, and its realization of art must have the participation of users.

At present, we think it is better to understand the network art concept in broad sense. Because, the mature of any concept not only needs the practice test, but also needs more attention from different disciplines and different angles.

\section{Through the cultural transmission, realize network art cultural value}

Many Chinese youth cartoons can really resonate good things in the young generation. We should capture the positive effect of this phenomenon, but also overcome its negative aspects. In order to eliminate the negative effects, it needs a high level education guide. Teachers should hold a profound discussion of cultural aggression, so as to achieve the education function of non aesthetic. Learning the advantage of cartoon works from Japan, the United States and South Korea, integrating and promoting the Chinese culture at the same time, and carrying forward the traditional virtues of the Chinese nation, have a great positive significance to create a harmonious world. Carrying forward the Chinese nation's "harmony" spirit helps us to "upright support, evil equits", and makes our young generation to grow up healthily. ${ }^{[2]}$

China network works of art should meet the Chinese aesthetic appeal and aesthetic psychology, and should be related to the social reality of China, because if the works present a completely strange world for the participants, it will produce com- pletely frustrated psychological effect for the participants, and the works also could not reach predictable effect. Internet Art is a kind of experimental art or avantgarde art in essence, different former the face art (including paper art, architectural art, body art and so on), which exists in the network space, and is virtual and immaterial. Its emerge is the challenge to our traditional art programs or art forms. But there is not any gap between the network art and Chinese traditional art, and they have the opportunity of fusion. Their fusion can update the perceptual world of the viewers, and meet the aesthetic psychological needs of the viewers. ${ }^{[3]}$ As a new developmental form of art, the network art is included in the art system and must have the elements of art undoubtly. Chinese traditional art is the result of civilization and culture which is five thousand years and is a wealth of artistic treasures. Chinese network art could combine with the traditional art completely, and creates art works of more aesthetic effect. ${ }^{[4]}$

Network is a huge social dye vat, therefore network art will also be dyed various colors, even deteriorate. The rapid development of the society makes people much more utilitarianism and material, and the warmth between people are severely neglected. In many cases, if minors couldn't find warm and family affection in reality, they will go to the web to search it, and the modification of network art such as network eroticism and violence literatures, pictures, films and videos, may infringe the minors who often surf on the internet. As to the metamorphic or inferior network art, we must use the combination approach of "intercept and leading". That is to say, it should pay attention to take off, remove, restrictions and other measures, also use dredge guiding vent, influencing and educating the minors and adults with positive, progressive and excellent network art works of films and 
televisions. Network art education has the responsibility to avoid the vulgarization of sensory stimulation . ${ }^{[5]}$ The managers of internet or the teachers of network art have the responsibility to guide the students to appreciate the lofty, refined and solemn network arts, and stay away from the so called network art works of low tastes and sensual tease, achieving the noble sentiments of the mold and the sublimation of aesthetic realm, and make effective guide to the direction of "fine works", "magic works" and "elegant works".

\section{Through the school teaching, built network art education system}

In our country, the network art education has not entered the classroom basically. Although some teachers or individual schools have begun to join the network art content in the art education programs, a complete network art education curriculum and teaching system have not been reported. Therefore, it is necessary to start to offer "network art education" course as soon as possible in the school teaching course system. Especially in the middle and primary schools, we improve the existing art, music and other art courses, increasing the network art basic knowledge, basic theory and basic method. The important task of contemporary education is to be people-oriented and to improve the whole quality of social mainstream adult, including information technology operation ability and the information society understanding and grasping ability. The society and school should construct the "the Trinity" education network of society, school and family, strengthening and improving the construction work of minors' ideology, moral idealism and faith, and assisting the parents to complete the modern human growth process of minors knowledge acquisition and soul creation. ${ }^{[6]}$
In the school, as to the implementation of the network art education, we should avoid the simple technique tendency of slavery instrument, and avoid the pure theory indoctrination and preaching bias detaching the practical operation. It does not only need to take some software as the information technology knowledge which is necessary to learn, but also need to pay attention to training students' visual function, so that it can grasp the objective things creatively and completely structurally, and especially promote the special imagination and creativity. It's suggested to pay attention to the individual differences of students, teach students in accordance with their aptitude and fully develop their potential on the basis of a comprehensive understanding of students' personality characteristics. The most important is to guide students to study independently, and promote students' imagination and independent thinking ability. Guide the students to establish a correct evaluation of the aesthetic through appreciation and evaluation education of the network art works, deal with the relationship between inheritance and creative creation in composition, handle the relationship between the software tools and the artistic soul, handle the relationship between entertainment and elegant, and handle the relationship between the formal beauty and the spirit and cultural noble. Realize the creativity cultivation, character molding and soul creation of the recipients, with the aesthetic appeal of art. ${ }^{[7]}$

\section{Through the network art education to achieve the traditional art educa- tion innovation}

Compared with the traditional model of art education, network model of art education has the special value of multi-level and multi-perspective; therefore, it is likely to achieve the traditional art educa- 
tion innovation through the art education of network, embodied in the following two points:Firstly, the network art can realize the huge expansion of the artistic imagination. ${ }^{[8]}$ Secondly, the network art can achieve works copied infinitely and the audience roaming freely.

In an art education of network mode, a large number of precious and rare art treasures can be easily copied through the network art, replacing the only existence and value of the original one, and making the recipient watching at any time in his own environment, which makes replication objects generate new vitality. From one perspective, it can't be achieved by the art education of traditional model. ${ }^{[9]}$

The major difference between network space and real space is that network time can be instantly passed through by just one click. This is not possible in the real space. ${ }^{[10]}$ Any work of art in the real time are fixed at a certain time, and it is very difficult to achieve the link within one second. But network art can easily achieve this. A hypertext link is not only very easy, but also lures people to continue clicking and browsing, finally realizing the free roaming. Even the roaming is treated as purpose, and thinking is took place by behavior, roaming becoming an art itself. ${ }^{[1]}$

\section{Acknowledgment}

This paper receives the following projects funded: Hebei Social Sciences Fund Project "Hebei public service-oriented egovernment Web2.0"; Qinhuangdao Social Science Key applied research topic In 2012 "On the construction of the Qinhuangdao-digital-city"and "On the construction of the Qinhuangdaoecological-city".

\section{Reference}

[1] Ying Chen. Network art and audience's aesthetic needs. Wuhan University of Technology: 15-18(2006).

[2][5][6][7] Yiwei Peng, Chunguang Wang. The network art education development problem analysis and countermeasure. Liaoning Education Research, (5):37-39(2008).

[3] Yi Zhang. Network art and Chinese traditional art. Journal of Huaiyin Normal University (Philosophy and Social Sciences Edition), (2) :260262(2007).

[4] Yi Zhang. On the harmony of the network art and Chinese traditional art . The Qilu art gallery, ( 6 ) :8891(2006).

[8] Yufang Bai. The paradox of network art education. Success (Education), (4) :106-107(2007).

[9] Jiangluquan, HuDaiping, ChiZhongren.Study on Frame of manoriented e-government of community. Forum of Post doctorate of ShangHai in 2005,Electronic Information:pp445-452(2005).

[10] Liu Bangfan; Zhang Jing; Liu Naixi . On the service as a logical thing.Proceedings of 2011 IEEE International Conference on Service Operations, Logistics and Informatics, SOLI 2011, p 182-185, 2011

[11] Liu Bangfan; Jiang Nan; Xu Shui .On the application of fuzzy logic in the computer engineering and management engineering. 2011 2nd International Conference on Artificial Intelligence, Management Science and Electronic Commerce, AIMSEC 2011 - Proceedings, p 1271-1273, 2011 